\title{
Effect of the temperature of polyurethane dissolution on the mechanism of wet-casting membrane formation
}

\author{
Liao-Ping Cheng ${ }^{a}$, Yen-Shih Huang ${ }^{\text {, }}$, Tai-Horng Young ${ }^{b, *}$ \\ a Department of Chemical Engineering, Tamkang University, Taipei 25137, Taiwan, ROC \\ ${ }^{\mathrm{b}}$ Institute of Biomedical Engineering, College of Medicine and College of Engineering, National Taiwan University, \\ Taipei 10016, Taiwan, ROC
}

Received 22 February 2002; received in revised form 5 August 2002; accepted 4 September 2002

\begin{abstract}
An analysis of data from scanning electron microscopy (SEM), gel permeation chromatography (GPC) and differential scanning calorimetry (DSC) for two series of polyurethane membranes is presented. Membranes were prepared by direct immersion of the casting solution of polyurethane, dissolved in dimethyl formamide at different temperatures, into either water or 1-octanol bath at $25^{\circ} \mathrm{C}$. Depending on the temperature of polyurethane dissolution, a gradual variety in the membrane structure was observed by SEM. As the temperature of polymer dissolution was increased, the membrane structure changed from dense to cellular or particulate morphologies. On the basis of the GPC and DSC results, polyurethane molecular weight decreased but the degree of microphase separation caused by clustering of some of the soft and hard segments into separate domains in the membrane increased with increasing the temperature of polymer dissolution. It is thus proposed that the change in membrane structure is due to the variation of molecular weight of polyurethane, which in turn can change polymer chain mobility during membrane formation. In addition, the extent of microphase separation was described and related to the particulate morphology when 1-octanol was used as the nonsolvent.
\end{abstract}

(c) 2002 Elsevier Science Ltd. All rights reserved.

Keywords: Polyurethane; Dissolution temperature; Particulate membranes

\section{Introduction}

Recently polymeric membranes have achieved commercial importance in a variety of separation applications [1]. Nonsolvent-induced phase inversion process is one of the most widely used means of preparing polymeric membranes [2]. In this process, a polymer solution is brought into contact with nonsolvent to induce phase separation, which forms the membrane. Polymer dissolution is a slow process so dissolving polymer in solvent is usually speeded by agitation. However, polymer

\footnotetext{
${ }^{*}$ Corresponding author. Tel.: +886-2-23123456x1455; fax: +886-2-23940049.

E-mail address: thyoung@ha.mc.ntu.edu.tw (T.-H. Young).
}

dissolution process may be still quite slow for polymers with high molecular weight and at high concentration. Alternative approach is to increase the dissolution temperature to enhance rate of polymer dissolution. In this work, the relationship between the temperature of polyurethane dissolution and the membrane structure was studied. Polyurethane used in this study was the commercial Pellethane 2103-80AE (Dow Chemical Company), based on $4,4^{\prime}$-methylene bis (phenylene isocyanate) (MDI), polytetramethylene oxide (PTMO) and 1,4 butanediol. Membranes were prepared by direct immersion of polyurethane solution into either water or 1-octanol bath. The prepared membranes were analyzed by scanning electron microscopy (SEM), gel permeation chromatography (GPC) and differential scanning calorimetry (DSC) to elucidate the membrane formation mechanism. 
Polyurethanes are a large family of synthetic polymers that are composed of alternating soft polyether segments, most commonly long linear polyethers, and hard urethane segments consisting of urethane linkages and aromatic groups. The existence of microphase separation, caused by clustering of some of the hard and soft segments into separate domains, has been well established [3]. The excellent mechanical properties of polyurethanes can be ascribed to the coexistence of these domains. While polyurethanes are susceptible to thermal, chemical and hydrolytic degradation [4], they may undergo different degrees of degradation in a given solvent at high temperature. Therefore, when the temperature of polymer dissolution is increased, polyurethane can dissolve more readily but this effect may decrease its molecular weight to some degree. In addition, Matsumoto et al. [5] have proposed that the polymer molecular weight can influence the poly (ethylene-co-vinyl alcohol) (EVAL) membrane structure. Membranes prepared from high molecular weight EVAL had large closed pores and a relatively lower number of pores, while those prepared from low molecular weight EVAL yielded small open pores and a large number of pores. Hence, the purpose of this paper was to examine whether polyurethane molecular weight degraded at higher dissolution temperature to result in the change of the membrane structure. We found the membrane structure changed from dense to cellular or particulate morphologies when the temperature of polymer dissolution was increased. Recently, particulate membranes have been explored as an alternative to various application of replacing traditional dense, porous or asymmetrical membranes in our laboratory [6-10]. Different particulate membranes have proved useful in microfiltration [6,7], plasma protein separation [8,9], and cell culture [10]. Therefore, this paper provides an easy method to prepare polyurethane membranes with the particulate morphology. Furthermore, the extent of microphase separation caused by clustering of hard and soft segments was described and related to the particulate morphology.

\section{Experimental}

\subsection{Membrane preparation}

Polyurethane (Pellethane 2103-80AE) membranes were prepared using direct immersion precipitation method. A homogeneous dope composed of $3 \mathrm{~g}$ of Pellethane elastomers and $7 \mathrm{~g}$ of dimethyl formamide (DMF) was spread uniformly on a glass plate to form a laminate $(\approx 250 \mu \mathrm{m})$ which was then immersed immediately into water or 1-octanol to effect polymer precipitation. In this work, dopes were prepared by dissolving Pellethane at 60,90 and $120{ }^{\circ} \mathrm{C}$, respectively, for 3 days and then kept at $25^{\circ} \mathrm{C}$ for 1 day. These dopes were commonly observed to precipitate upon standing, thus, they were heated to $60{ }^{\circ} \mathrm{C}$ to ensure complete dissolution before immersing into the precipitation bath at $25{ }^{\circ} \mathrm{C}$. The precipitation time required for water and 1-octanol were $1 \mathrm{~min}$ and $1 \mathrm{~h}$, respectively. The residual solvent was removed from the membrane by a series of washing steps. Finally, the formed membrane was dried in open air.

\subsection{Membrane characterization}

The morphology of the membrane was examined in different views by using SEM. The samples were sputtered with gold and palladium in a vacuum and using a Hitachi S-800 microscope at $20 \mathrm{kV}$.

To evaluate the effect of polymer dissolution temperature on thermal degradation of polyurethane, the membranes were analyzed by GPC using the PLgel $5 \mu \mathrm{m}$ mixed-D column from Polymer Lab., UK. The prepared membranes were dissolved in tetrahydrofuran (THF) in the concentration of $0.5 \%$ by weight. Ultra pure-grade THF (Alps Chem., Taiwan) was used as the mobile phase. The effluent flow rate was maintained at $1.0 \mathrm{ml} /$ min using a SPECTROFLOW 400 pump (Applied Biosystem Corporation, USA). The refractive index detector in this GPC system was Shodex RI SE-61 (Showa Denko Corporation, Japan). Monodisperse polystyrene standards dissolved in THF were used to calibrate the system.

The thermal transition behavior of each membrane was studied using a sensitive DSC 2010 (TA instruments Ltd., USA) to understand the degree of microphase separation in the polyurethanes. DSC was calibrated by using indium as standards. The measurements were performed under a nitrogen atmosphere at a heating rate of $10{ }^{\circ} \mathrm{C} / \mathrm{min}$ to observe the glassy transition temperature and characteristic endothermic transition. The characteristic endothermic transition is taken as the temperature at the endothermic peak of calorimetric transition.

\section{Results}

\subsection{SEM analysis}

Fig. 1 shows the top and cross-sectional views of polyurethane membranes, which was prepared by immersing the polyurethane solution dissolved in DMF at different temperatures into water. In the cross-sectional photographs, upper side corresponds to the top surface. When polyurethane was dissolved at $60{ }^{\circ} \mathrm{C}$, there were 

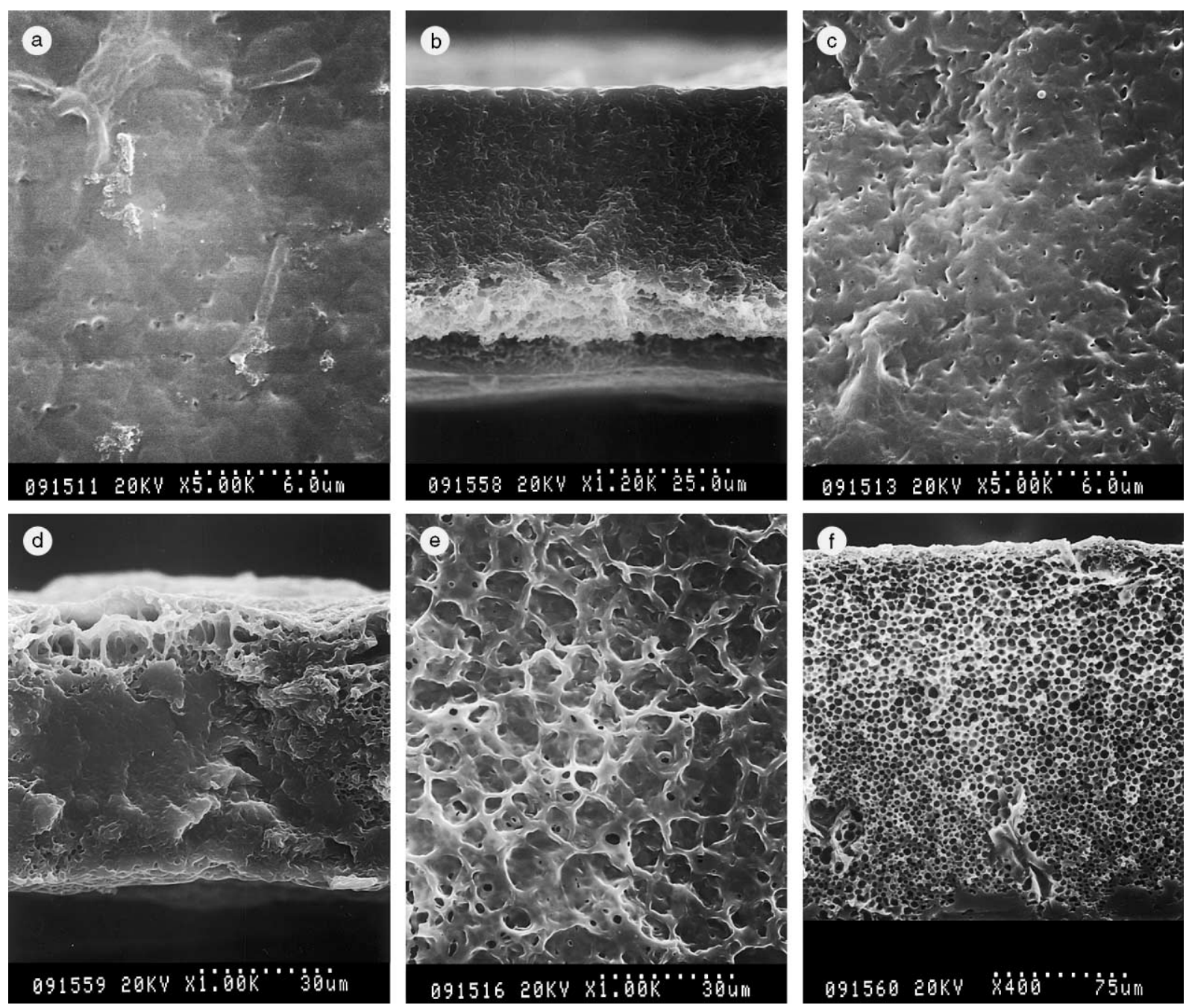

Fig. 1. SEM photomicrographs of polyurethane membranes prepared by immersing the polyurethane solution dissolved at different temperatures into water: (a) $60{ }^{\circ} \mathrm{C}$, top surface; (b) $60^{\circ} \mathrm{C}$, cross section; (c) $90{ }^{\circ} \mathrm{C}$, top surface; (d) $90{ }^{\circ} \mathrm{C}$, cross section; (e) $120{ }^{\circ} \mathrm{C}$, top surface; (f) $120^{\circ} \mathrm{C}$, cross section.

few pores like the artifact of a rough and sunken surface on the membrane surface (Fig. 1(a)) but no porous structure was observed in the cross-sectional photograph (Fig. 1(b)). Therefore, the whole structure in this case is regarded as a dense morphology. On the other hand, when the polyurethane dissolution temperature was increased to $120{ }^{\circ} \mathrm{C}$, the porous structure was formed in the membrane surface (Fig. 1(e)) and its cross section consisted of cellular pores enclosed in the continuous polymer matrix phase (Fig. 1(f)). These pores were separated and had the nearly same size (ca. several $\mu \mathrm{m}$ ). Such a porous configuration is similar to the so-called "sponge structure" that are produced by the liquidliquid demixing process. The structure of the membrane prepared by dissolving polyurethane at $90{ }^{\circ} \mathrm{C}$ is shown in Fig. 1(c) and (d). A rough surface and an irregular cross section with some pores were observed. Such a structure represents the intermediate case between membranes prepared by dissolving polyurethane at 60 and $120^{\circ} \mathrm{C}$.

Fig. 2 presents the top and cross-sectional views of polyurethane membranes prepared by immersing the polyurethane solution dissolved in DMF at different temperatures into 1-octanol. When polyurethane was dissolved at $60{ }^{\circ} \mathrm{C}$, the membrane surface obviously had many spherical particles (Fig. 2(a)), which was supported by a thick dense sublayer (Fig. 2(b)). Besides few pores existing near the membrane surface, there was almost not any pores or particles existing in the cross section. When the temperature of polymer dissolution 

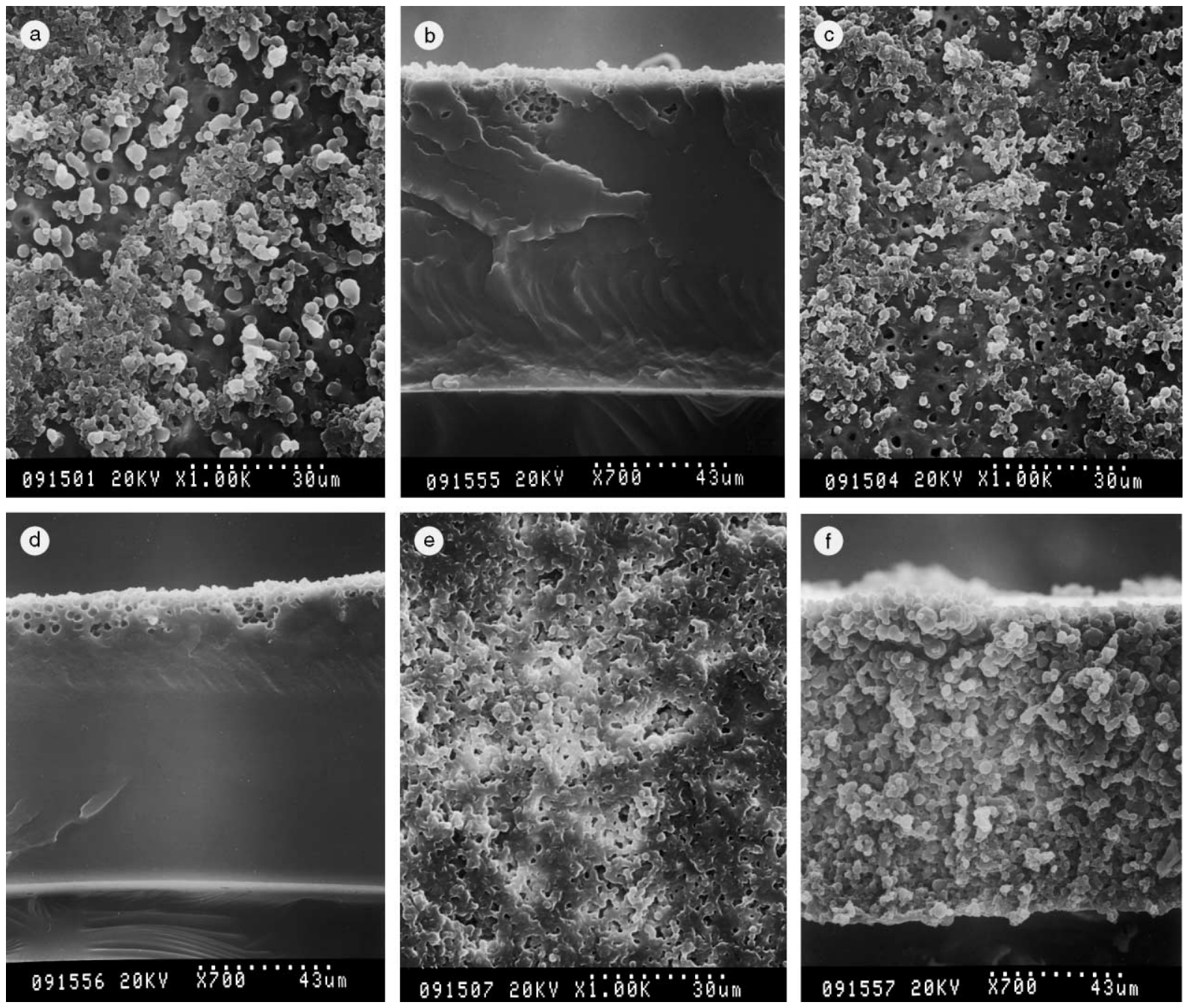

Fig. 2. SEM photomicrographs of polyurethane membranes prepared by immersing the polyurethane solution dissolved at different temperatures into 1-octanol: (a) $60{ }^{\circ} \mathrm{C}$, top surface; (b) $60{ }^{\circ} \mathrm{C}$, cross section; (c) $90^{\circ} \mathrm{C}$, top surface; (d) $90{ }^{\circ} \mathrm{C}$, cross section; (e) $120{ }^{\circ} \mathrm{C}$, top surface; (f) $120^{\circ} \mathrm{C}$, cross section.

was increased to $90^{\circ} \mathrm{C}$, the membrane surface and cross section (Fig. 2(c) and (d)) were still similar to those of membranes prepared by dissolving polyurethane at $60{ }^{\circ} \mathrm{C}$. However, when the polyurethane dissolution temperature was increased to $120^{\circ} \mathrm{C}$, spherical particles existed not only on the membrane surface (Fig. 2(e)) but also throughout the cross section (Fig. 2(f)). Although the particles were difficult to distinguish each other due to incomplete coalescence of particles, the size of the particles approximately fell in the range of a few micrometers. The mechanical strength of particulate membranes depends heavily upon the links between polymer particles. Since the linkage points are relatively limited, only on the interface between spheri- cal particles, their strength is not that satisfactory as membranes with cellular pores enclosed in the continuous polymer matrix phase. However, the mechanical strength of the particulate membrane is still strong enough to various applications [6-10]. In addition, such porous and open structure can fulfill the requirement of scaffolds to maximize cell adhesion, growth and differentiation in tissue engineering applications. Therefore, in our laboratory, particulate membranes are used as a supporting material to act as a scaffold for cell growth. We have previously demonstrated that osteoblast cells cultured on particulate surfaces were well spread and flattened, and had a great number of adhesion [11]. 


\subsection{GPC analysis}

Table 1 shows the weight average molecular weight and molecular weight distribution of prepared polyurethane membranes. The data reveals the weight average molecular weight of polyurethane decreased as the dissolution temperature was increased. Grassie and $\mathrm{Zu}-$ lfiqar claimed that the thermal degradation of polyurethanes is primarily a depolycondensation process, namely the reverse of the reaction in which they are formed [12]. Based on the present result, polyurethane degradation was greatly influenced by the dissolution temperature, which might further influence the membrane formation mechanism (discussed below). In addition, the polydispersity of polyurethane also shows a decreasing trend. This can be ascribed to the fact that the low molecular weight fragments degraded at higher dissolution temperature were removed during membrane formation. Therefore, the loss of low molecular weight fragments should have more impact on the weight average molecular weight and decreased molecular weight distribution.

\subsection{DSC analysis}

Fig. 3 shows the DSC thermograms collected from polyurethane membranes prepared by immersing the polyurethane solution dissolved in DMF at $60{ }^{\circ} \mathrm{C}$ into water and 1-octanol. Briefly, the typical glass transition temperature $\left(T_{\mathrm{g}}\right)$ of the soft segment around $-50{ }^{\circ} \mathrm{C}$ and a broad peak with a maximum around $150{ }^{\circ} \mathrm{C}$ were found. This broad peak present in the temperature range $70-150{ }^{\circ} \mathrm{C}$ can be considered as a merged endotherm from two peaks at approximately 70 and $150{ }^{\circ} \mathrm{C}$, which are due to the disruption of short and long range order of hard segments, respectively, as proposed by Seymour and Cooper [3]. Similar DSC curves were obtained for all polyurethane membranes studied (not shown here).

The $T_{\mathrm{g}}$ of the soft segment and the peak temperature of merged endotherm for all prepared polyurethane membranes are summarized in Table 2. The position of

Table 1

Weight average molecular weight and molecular weight distribution of prepared polyurethane membranes

\begin{tabular}{lrl}
\hline $\begin{array}{l}\text { Polyurethane dissolution } \\
\text { temperature }\left({ }^{\circ} \mathrm{C}\right)^{\mathrm{a}}\end{array}$ & $M_{\mathrm{w}}(\mathrm{g})^{\mathrm{b}}$ & $\mathrm{MWD}^{\mathrm{c}}$ \\
\hline 60 & 359,000 & 2.24 \\
90 & 237,000 & 2.04 \\
120 & 97,900 & 1.93 \\
\hline
\end{tabular}

${ }^{\mathrm{a}}$ The change of molecular weight of polyurethane membranes was independent of the nonsolvent so dependent on the polyurethane dissolution temperature only.

${ }^{\mathrm{b}} M_{\mathrm{w}}$ : weight average molecular weight.

${ }^{\mathrm{c}}$ MWD: molecular weight distribution.

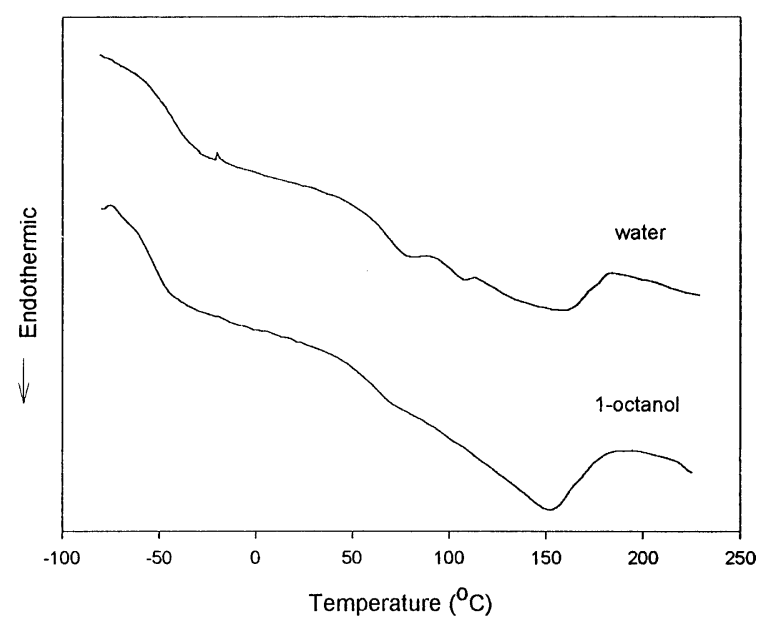

Fig. 3. DSC thermograms from polyurethane membranes prepared by immersing the polyurethane solution dissolved at $60{ }^{\circ} \mathrm{C}$ into water and 1 -octanol.

Table 2

DSC first heat data for polyurethane membranes

\begin{tabular}{lcll}
\hline $\begin{array}{l}\text { Precipitation } \\
\text { medium }\end{array}$ & $\begin{array}{l}\text { Polyurethane } \\
\text { dissolution } \\
\text { temperature } \\
\left({ }^{\circ} \mathrm{C}\right)\end{array}$ & $\begin{array}{l}\text { Glass transi- } \\
\text { tion temper- } \\
\text { ature of the } \\
\text { soft segment } \\
\left({ }^{\circ} \mathrm{C}\right)\end{array}$ & $\begin{array}{l}\text { Peak temper- } \\
\text { ature of the } \\
\text { merged } \\
\text { endotherm } \\
\left({ }^{\circ} \mathrm{C}\right)\end{array}$ \\
\hline Water & 60 & -45.4 & 157.7 \\
Water & 90 & -49.7 & 158.8 \\
Water & 120 & -56.7 & 146.0 \\
1-Octanol & 60 & -53.1 & 151.4 \\
1-Octanol & 90 & -59.7 & 150.5 \\
1-Octanol & 120 & -60.7 & 150.8 \\
\hline
\end{tabular}

peak temperature of the merged endotherm presents no discernible quantitative trend but the $T_{\mathrm{g}}$ of the soft segment shows the polymer dissolution temperature could modify the state of microphase separation. When water was used as the nonsolvent, $T_{\mathrm{g}}$ decreased from -45.4 to $-56.7{ }^{\circ} \mathrm{C}$ with increasing the polyurethane dissolution temperature from 60 to $120{ }^{\circ} \mathrm{C}$, which is substantially higher than that of pure soft-segment polymer. (The $T_{\mathrm{g}}$ of poly (tetramethylene oxide) is about $-82{ }^{\circ} \mathrm{C}$ [13].) This indicates a certain degree of hard and soft segment mixing. Conversely, the polyurethane membranes with using 1-octanol as the nonsolvent exhibited slight lower $T_{\mathrm{g}}$ of -53.1 to $-60.7^{\circ} \mathrm{C}$, which appears that hard segment solubilization in the soft segment matrix is smaller. This indicates an improved microphase separation when 1-octanol was used as the nonsolvent to prepare polyurethane membranes. Furthermore, polyurethane membranes exhibited a decrease of $T_{\mathrm{g}}$ by 7 to $11^{\circ} \mathrm{C}$ when the polymer dissolution temperature was increased, irrespective of immersing polyurethane solution into water or 1-octanol. This 
suggests polyurethane membranes consist of higher purity of the soft segment phase when the polymer dissolution temperature was increased. This is probably caused by the fact that the soft segments and the hard segments could organize the membrane microstructure in the course of preparation of the membrane. However, this process will be impeded in the case of polymer with higher molecular weight due to the serious molecular entanglements. Therefore, polyurethane molecules with low molecular weight in the casting solution can reorganize the membrane microstructure during membrane formation.

\section{Discussion}

The formation of porous structure in a membrane is dictated by the sequence of the phase transition events in the precipitation process $[14,15]$. Many parameters, such as the compositions of the casting solution and the precipitation bath, and the temperature of the system, are known to exert certain influences on the morphology of the formed membranes [1,2]. In this study, depending on the polyurethane dissolution temperature, a gradual variety in membrane structures could be observed via SEM. In addition, two different types of membranes were synthesized in two nonsolvent-DMF-polyurethane systems. At low dissolution temperatures (e.g., $60{ }^{\circ} \mathrm{C}$ ), phase separation of polyurethane solution by exchange of solvent and nonsolvent was clearly limited, which forms the dense structure. Whereas at elevated dissolution temperatures (e.g., $120^{\circ} \mathrm{C}$ ) liquid-liquid demixing process dominated the precipitation process by using water as the nonsolvent and the membrane became cellular in its morphology. As the nonsolvent using 1octanol, the membrane showed a homogeneous particulate morphology while the cellular morphology was suppressed.

It is desirable to characterize the differences in morphology of the two series of polyurethane membranes. Since membrane preparation is a nonequilibrium process, this clearly implies that the change in membrane structure is attributed to the arrangement of polymer chains during membrane formation. When casting solutions containing high molecular weight polymer are immersed in a coagulation bath, the molecular entanglements of polymer should restrict chain mobility. Therefore, polymer molecules are brought rapidly into a state wherein vitrification takes place and the structure is frozen. Indeed, GPC studies suggested that the polyurethane dissolution temperature had pronounced effect on the polyurethane degradation, as indicated in Table 1. Therefore, at low dissolution temperatures (e.g., 60 ${ }^{\circ} \mathrm{C}$ ), polyurethane molecules tended to aggregate to form a dense structure by virtue of a great number of molecular entanglements. This membrane was very hard and stiff during membrane formation so as to decrease the possibility of microporous regions besides the membrane surface. Conversely, as the molecular weight was decreased at elevated dissolution temperatures (e.g., $120{ }^{\circ} \mathrm{C}$ ), polyurethane molecules with sufficient mobility in the membrane solution can arrange themselves faster than the nonsolvent-induced polymer vitrification. Hence, polymer molecules underwent liquid-liquid demixing or solid-liquid demixing according to the thermodynamic equilibrium. Consequently, the structure of the polyurethane membranes did not only depend on the dissolution temperature but also varied with the nonsolvents used.

Another interesting feature of polyurethane membranes is that they can form a symmetric structure by constituent particles bonded to each other when 1-octanol was used as the nonsolvent. It is known that many investigators have prepared different polymer membranes with similar particulate morphology by using 1octanol as the nonsolvent though these polymers have rather different chemical properties $[8,16-18]$. With different circumstances, there can be two possible mechanisms for the formation of particles in membranes. The first possibility is that particles are generated by the spinodal demixing [19]. The second possible origin of particles is crystallization of polymer during the membrane formation process $[14,20,21]$. Since the building particles in our polyurethane membranes are greater than the size of nodules caused by the spinodal demixing, it is suggested that particle structure cannot be ascribed to the spinodal demixing. However, it is also not certain at this point whether the particle structure in our polyurethane membranes is generated by crystallization of polyurethane. Polyurethane membranes may be considered as soft segments dispersed within the hard domains and as hard segments dispersed in the soft matrix [3]. Thus, it is reasonable to suggest the particle structure can be attributed to an improvement in the extent of microphase separation in polyurethane membranes with 1octanol as the nonsolvent. Indeed, DSC studies show that polyurethane membranes prepared by using 1-octanol as the nonsolvent had a well-phase-separated morphology, a lower value in $T_{\mathrm{g}}$ of the soft segment. The decrease of $T_{\mathrm{g}}$ could be explained by assuming that the rearrangement of polymer chains during the precipitation process produces a decrease of the percent of soft segments dissolved in the hard segments phase. For these reasons, we propose that locus of particle nucleus resulted from microphase separated polymers, which is beneficial to the chains to pack into a regular lattice. It seems logical that the small initially formed particles needed better chain mobility and longer induction time. This is consistent with the observed longer precipitation time for low molecular weight polyurethane membranes to present a symmetric structure by constituent particles bonded to each other. 
In summary, the exact nature of particles has not yet been satisfactorily resolved but it should be noted that polyurethane membranes prepared from using 1-octanol as the nonsolvent contained a well-phase-separated morphology, suggesting that nonsolvent-induced ordering played an important role during membrane formation. Consequently, the complex membrane structures can be ascribed to various levels of packing order in the soft segment and hard segment domains.

\section{Acknowledgements}

This work is supported by the National Science Council of the Republic of China.

\section{References}

[1] Mulder M. Basic principles of membrane technology. Dordrecht, The Netherlands: Kluwer; 1991.

[2] Kesting RE. Synthetic polymeric membranes. New York: John Wiley and Sons; 1985.

[3] Seymour RW, Cooper SL. Thermal analysis of polyurethane block polymers. Macromolecules 1973;6:48-53.

[4] Lelah MD, Cooper SL. Polyurethanes in medicine. Boca Raton: CRC Press; 1984.

[5] Matsumoto T, Nakamae K, Ochiumi T, Horie S. Effect of molecular weight of ethylene vinyl alcohol copolymer on membrane structures. J Membr Sci 1981;9:109-19.

[6] Cheng LP, Lin HY, Chen LW, Young TH. Solute rejection of dextran by EVAL membranes with asymmetric and particulate morphologies. Polymer 1998;39:2135-42.

[7] Young TH, Cheng LP, Lin HY. Interesting behavior for filtration of macromolecules through EVAL membranes. Polymer 2000;41:377-83.

[8] Lin DT, Cheng LP, Kang YJ, Chen LW, Young TH. Effects of precipitation conditions on the membrane morphology and permeation characteristics. J Membr Sci 1998;140:185-94.

[9] Young TH, Lin DT, Chen LY, Huang YH, Chiu WY. Membranes with a particulate morphology prepared by a dry-wet casting process. Polymer 1999;40:5257-64.
[10] Young TH, Yao CH, Sun JS, Lai CP, Chen LW. The effect of morphology variety of EVAL membranes on the behavior of myoblasts in vitro. Biomaterials 1998;19:71724.

[11] Wang JH, Yao CH, Chuang WY, Young TH. Development of biodegradable polyesterurethane membranes with different surface morphologies for the culture of osteoblasts. J Biomed Mater Res 2000;51:761-70.

[12] Grassie N, Zulfiqar M. Thermal degradation of the polyurethane from 1,4-butanediol and methylene bis(4phenyl isocyanate). J Polym Sci, Polym Chem Ed 1978;16: 1563-74.

[13] Wang CB, Cooper SL. Morphology and properties of segmented polyether polyurethaneureas. Macromolecules 1983;16:775-86.

[14] Young TH, Lai JY, Yu WM, Cheng LP. Equilibrium phase behavior of the membrane forming water-DMSOEVAL copolymer system. J Membr Sci 1997;128:55-65.

[15] Young TH, Hsieh CC, Chen LY, Huang YS. The formation mechanism of membranes prepared from the crystalline EVAL polymer-water (nonsolvent)-2-propanol (nonsolvent) system. J Membr Sci 1999;159:21-7.

[16] Broens L, Koenhen DM, Smolders CA. On the mechanism of formation of asymmetric ultra- and hyper-filtration membranes. Desalination 1977;22:205-19.

[17] Wijmans JG, Rutten HJJ, Smolders CA. Phase separation phenomena in solutions of poly(2,6-dimethyl-1,4-phenyleneoxide) in mixtures of trichloroethylene, 1-octanol, and methanol: relationship to membrane formation. J Polym Sci, Polym Phys 1985;23:1941-55.

[18] Burghardt WR, Yilmaz L, McHugh AJ. Glass transition, crystallization and thermoreversible gelation in ternary PPO solution; relationship to asymmetric formation. Polymer 1987;28:2085-92.

[19] Nunes SP, Inoue T. Evidence for spinodal decomposition and nucleation and growth mechanisms during membrane formation. J Membr Sci 1996;111:93-103.

[20] Bulte AMW, Folkers B, Mulder MHV, Smolders CA. Membranes of semicrystalline aliphatic polyamide nylon 4,6: formation by diffusion-induced phase separation. J Appl Polym Sci 1993;50:13-26.

[21] Cheng LP, Dwan AW, Gryte CC. Membrane formation by isothermal precipitation in polyamide-formic acid-water systems. II. Precipitation dynamics. J Polym Sci, Polym Phys 1995;33:223-35. 